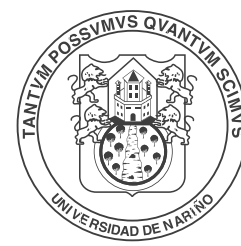

REVISTA DE CIENCIAS AGRÍCOLAS

RESEARCH ARTICLES

Volumen 32(2):104 - 112

Segundo Semestre

ENTOMOLOGIA AGRÍCOLA

ISSN Impreso 0120-0135

\title{
Respuesta fisiológica del ñame espino (Dioscorea Rotundata Poir) a las densidades de siembra
}

Physiological response of the white yam (Dioscorea rotundata Poir) to planting densities

Dairo J. Pérez ${ }^{1 *}$; Rodrigo 0. Campo ${ }^{2}$; Alfredo Jarma $0^{2}$.

\footnotetext{
${ }^{1}$ M.Sc. Docente Catedrático. Universidad de Córdoba, Departamento, Montería, Colombia, dairoperez81@hotmail.com.

${ }^{2}$ Ph.D. Docente Titular. Universidad de Córdoba, Montería, Colombia.
}

Citar: PÉREZ D.; CAMPO, R.; JARMA, A. 2015. Respuesta fisiológica del ñame espino (Dioscorea Rotundata Poir) a las densidades de siembra. Rev. Cienc. Agr. 32(2):104 - 112.

Fecha de recepción: Abril 10 de 2015

Fecha de aceptación: Noviembre 11 de 2015

\section{RESUMEN}

Esta investigación tuvo como objetivo estudiar la respuesta fisiológica del ñame espino (Dioscorea rotundata cv. brasilero) en función del incremento de las densidades de siembra, bajo la oferta ambiental del Sinú medio. El estudio se llevó a cabo en la Universidad de Córdoba (Montería, Colombia), donde se evaluaron las densidades de siembra de 45.455, 30.303, 22.727 y 10.101 plantas/ha; bajo un diseño de Bloques Completos al Azar con tres repeticiones. Las variables de respuestas consideradas fueron la fotosíntesis neta (Pn), medida en tres etapas fenológicas (vegetativa, inicio y final de tuberización); y los índices de crecimiento: tasa absoluta de crecimiento (TAC), tasa de asimilación neta (TAN), tasa relativa de crecimiento (TRC) e índice de área foliar (IAF). La Pn no varió entre las densidades de siembra $(p \leq 0,05)$, pero después de la etapa vegetativa, se redujo en promedio un 19,14\% para el inicio y final de la tuberización. La TAC y la TRC tuvieron similar tendencia, se incrementaron desde la etapa vegetativa, pero esta última se redujo al final del cultivo. La TAN comenzó a decrecer desde la etapa vegetativa con un leve incremento al final del ciclo. El IAF se incrementó significativamente a partir de la tuberización, para las densidades de siembra superiores a 10.101 plantas/ha, alcanzando valores entre 3,08 y 4,03. Se concluye que los índices de crecimiento a excepción del IAF y la Pn, no variaron entre las densidades de siembra, probablemente por la capacidad de la especie de regular su actividad fisiológica en función de la densidad de siembra.

Palabras clave: Fisiología de cultivos, tuberización, índices de crecimiento, intercambio gaseoso, fotosíntesis neta. 


\begin{abstract}
This research aimed to study the physiological response of the white yam (Dioscorea rotundata cv. brasilero) in relation to increasing planting densities under environmental conditions of the mid-Sinú region. This study was conducted at the Universidad de Córdoba (Montería, Colombia), there four planting densities: $\mathrm{f} 45.455,30.303,22.727$, and 10.101 plants/ha were evaluated through a randomized complete block design with three repetitions. The response variables assessed were net photosynthesis (Pn) -measured in three phenological stages (vegetative, start and end of tuberization)- and growth indices, such as absolute growth rate (AGR), net assimilation rate (NAR), relative growth rate (RGR), and leaf area index (LAI). The Pn did not vary between planting densities ( $p \leq 0.05$ ), but was reduced in average by $19.14 \%$ after the vegetative stage, for the start and end of tuberization. The AGR and RGR showed a similar tendency, with an increase in the vegetative stage, but a reduction towards the end of the cycle. The NAR began to increase in the vegetative stage, with a slight increase at the end of the cycle. The LAI increased significantly in tuberization for planting densities above 10.101 plants/ha, reaching values between 3.08 and 4.03. It is concluded that the growth indices evaluated, except for LAI and Pn, did not vary between planting densities, likely due to the species' capacity to regulate its physiological activity in relation to the planting density.
\end{abstract}

Keywords: Crop physiology, tuberization, growth indices, gas exchange, net photosynthesis.

\section{INTRODUCCIÓN}

El ñame (Dioscorea spp.) se caracteriza por la producción de tubérculos comestibles que hacen parte de la dieta alimenticia de la región Caribe, situación que explica su gran demanda en el mercado regional; sin embargo, en la actualidad se están exportando 2.654t a Estados Unidos,que generan divisas de hasta US \$ 2.711.000 (CNP, 2015).Las especies de mayor importancia en Colombia por área sembrada y demanda son Dioscorea alata y Dioscorea rotundata (Álvarez, 2000).

Según la FA0 (2013), Colombia es el tercer productor de ñame del continente americano, con una participación del 20,56\%, después de Haití (23,71\%) y Cuba (21,54\%). La producción nacional (314.991 t/año) proviene principalmente de la región Caribe, la cual, representa la principal fuente de seguridad alimentaria e ingresos de los pequeños productores, donde los departamentos de Bolívar, Córdoba y Sucre son los mayores productores con una participación del 44,24, 36,19 y 7,78\%, respectivamente (álvarez, 2000; Agronet, 2013).
En la región Caribe colombiana, este cultivo es desarrollado con tecnología tradicional, donde la densidad de siembra es una de las principales limitantes para el incremento de la producción y productividad. Los productores utilizan densidades de siembra de 10.000 plantas/ha que son consideradas bajas con referencia a las usadas por otros países como Costa Rica, que duplican o triplican su población (MAG, 1991; Sánchez y Hernández, 1998; Aguilar, 1998; Álvarez, 2000; Reina, 2012). Esta situación favorece la formación de tubérculos de gran tamaño, que son rechazados por el mercado internacional y ocasionan pérdidas significativas en postcosecha de hasta $30 \%$, obligando a los productores a realizar su venta en el mercado local con un menor precio.

La densidad de siembra define el máximo aprovechamiento de los recursos disponibles por la planta, en especial de la radiación solar, responsable del incremento de la fotosíntesis; la cual se traduce en una mayor acumulación y partición de biomasa hacia los tubérculos (Ballaré et al., 1995). Existen densidades de siembra recomendadas que solo consideran la producción 
y productividad, sin tener en cuenta la fisiología del cultivo. En este sentido, el MAG (1991) y Aguilar (1998), recomiendan establecer entre 22.222 y 33.333 plantas/ha en condiciones de suelo de Costa Rica. Por otra parte, bajo suelos de condiciones de Puerto Rico, Cardona (2007), recomienda la siembra de 54,649 plantas/ha en ñame espino (D. rotundata). Blake (1983), evaluó diferentes tamaños de semilla y espaciamientos de D. alata en suelos de condiciones de Jamaica, encontrando que la densidad de siembra óptima se encuentra entre 14,815 y 18,519 plantas/ha.

Según (Hall et al., 1993) el estudio de las tasas de fotosíntesis (Pn) es fundamental para describir la respuesta de fijación de Carbón en la hoja y el dosel de la plantas, frente a los factores ambientales. Mayores Pn indican mayor biomasa y producción de cultivos. Ekanayake y Jong (1992) encontraron en ñame bajo condiciones de Nigeria, altos valores de Pn $\left(9,08 \mu\right.$ moles de $\left.\mathrm{CO}_{2} \mathrm{~m}^{-2} \mathrm{~s}^{-1}\right)$ a partir de una radiación de $1364 \mu \mathrm{Em}^{-2} \mathrm{~s}^{-1}$. Por otra parte, Rodríguez y Leihner (1997) en D. alata reporta una Pn media de $1,36 \mu$ moles de $\mathrm{CO}_{2} \mathrm{~m}^{-2} \mathrm{~s}^{-1}$ con un rango entre 1,79 y $5,28 \mu$ moles de $\mathrm{CO}_{2} \mathrm{~m}^{-2} \mathrm{~s}^{-1}$. En investigaciones recientes, Liu y Zhong (2009) reportan en D. opposita bajo condiciones de China, valores de Pn que no superaron los $2,5 \mu$ moles de $\mathrm{CO}_{2} \mathrm{~m}^{-2} \mathrm{~s}^{-1}$ en plantas sometidas a radiación natural.

El estudio de los índices de crecimiento es fundamental para determinar el impacto de las variables ambientales sobre la producción de biomasa y su particionamiento en la planta (Hall et al., 1993). Rodríguez y Leihner (1997), reportan que la tasa absoluta de crecimiento (TAC) de D. alata bajo condiciones de Costa Rica se incrementó significativamente entre los 130 y 158 días después de la siembra, desde donde comienza a descender; pero la TAC de hojas decreció desde la tuberización. Por otra parte, según Melteras (2007), la tasa relativa de crecimiento (TRC) y la tasa de asimilación neta
(TAN) en D.esculenta comparten una máxima eficiencia desde las etapas iniciales hasta el desarrollo de la planta. El comportamiento de la TRC en D.esculenta coincide con los reportes de Rodríguez y Leihner (1997) en D. alata bajo la oferta ambiental de Costa Rica.

El estudio de la acumulación de biomasa, índices de crecimiento y tasas de fotosíntesis, ayudan a entender el efecto de la competencia intraespecífica sobre el rendimiento de los cultivos. Por ello, la presente investigación tuvo como objetivo estudiar la respuesta de la fotosíntesis (Pn) y del crecimiento de ñame espino (D.rotundata cv. brasilero) en función de incrementos de densidad de siembra bajo las condiciones de la oferta ambiental del Sinú medio.

\section{MATERIALES Y MÉTODOS}

Localización. El estudio se realizó en la Granja de Investigación de la Universidad de Córdoba, ubicada en el municipio de Montería a 14 msnm, 8 44' LN y $75^{\circ} 53^{\prime} \mathrm{LO}$. Esta zona se caracteriza por una precipitación media anual de 1346,1mm, humedad relativa del $84 \%$, temperatura media anual de $27,4^{\circ} \mathrm{C}$ y brillo solar anual de 2108,2 horas; condiciones que la clasifican como zona de bosque seco tropical (bs-T), según la clasificación de Holdrige (Palencia et al., 2006).

Material vegetal. Se utilizó el clon comercial de ñame espino (D. rotundata cv. Brasilero), el cual fue inicialmente propagado a partir de minisemillas (entre 50 y $60 \mathrm{~g}$ ) obtenidas de la sección superior 0 cabeza del tubérculo. Para garantizar la brotación completa y uniforme, se establecieron semilleros en arena, como sustrato inerte y esterilizado. Las plántulas fueron trasplantadas a las parcelas cuando los tallos alcanzaron una altura entre $15 \mathrm{y}$ $20 \mathrm{~cm}$ de altura. 
Manejo Agronómico. En las parcelas se realizaron todas las prácticas agronómicas que aplican los productores de la región Caribe. Cuando las plantas alcanzaron $60 \mathrm{~cm}$ de altura (estado de vareteo), fueron tutoradas en un sistema de espalderas, guiando su crecimiento con fibras plásticas sujetadas a un alambre.

Diseño experimental. Debido al efecto de la variabilidad de las condiciones edáficas, se estableció en campo un Diseño de Bloques Completamente al Azar (DBCA) con cuatro tratamientos y tres repeticiones. Cada parcela estuvo conformada por 50 plantas, distribuidas en cinco líneas de caballones separados a 1,1 m, sobre los cuales se sembraron 10 plantas, de acuerdo con la distancia de separación entre plantas de cada tratamiento. Los tratamientos fueron: 45.454, 30.303, 22.727 y 10.101 plantas/ha, donde la última densidad correspondió a la usada por el productor.

Las variables repuestas evaluadas fueron: fotosíntesis neta (Pn) y los índices de crecimiento (Hunt, 1982); tasa absoluta de crecimiento (TAC), tasa de asimilación neta (TAN), tasa relativa de crecimiento (TRC) e índice de área foliar (IAF).

La Pn fue medida mediante un analizador de gases al infrarrojo (IRGA) modelo CIRAS-2 PP System, programado con una radiación fotosintéticamente activa (RFA) de $1000 \mu$ moles de fotones $\mathrm{m}^{-2} \mathrm{~s}^{-1} \mathrm{y}$ concentración de $350 \mathrm{ppm}$ de $\mathrm{CO}_{2}$. En cada tratamiento se tomó al azar una hoja activa de la parte superior del dosel, ubicada en plantas de los dos surcos centrales y en plena competencia para hacer las mediciones entre 9:00 y 11:00 a.m.

Los índices de crecimiento fueron estimados a partir del área foliar y la materia seca. Para ello, en cada parcela se realizaron muestreos destructivos cada mes a partir del trasplante, tomando al azar dos plantas por parcela (Campo et al., 2012). En las muestras se estimó el área foliar, a través de la relación peso: área, registrando su peso en fresco y el peso de 10 círculos cortados con un sacabocado de área conocida $\left(0,2826 \mathrm{~cm}^{2}\right)$ y la materia seca mediante la deshidratación de las muestras en estufa de secado por 72 horas a $70^{\circ} \mathrm{C}$.

Análisis de datos. Los datos fueron sometidos a Análisis de Varianza (ANDEVA), del cual se verificó el cumplimiento de los supuestos; así mismo, se realizaron pruebas de comparación de medias de Tukey (5\%), empleando el programa estadístico SAS (Statistical Analysis System) versión 9.1.Solamente para la Pn, el ANDEVA se realizó mediante DBCA, en arreglo factorial de 3x4 (etapas fenológicas $\mathrm{x}$ distancias de siembra) con el fin de considerar como fuentes de variación las densidades y las etapas fenológicas (vegetativa, inicio tuberización y final de tuberización) y su interacción.

Para los índices de crecimiento el ANDEVA se realizó al inicio de la tuberización, esto, debido a que en este momento, teóricamente se marca el máximo incremento de biomasa de la planta y una de las máximas Pn. Después de verificar el cumplimiento de los supuestos del ANDEVA fue necesaria una transformación logarítmica $(\mathrm{Y}+1)$ para el IAF y la prueba no para métrica de rangos de Friedman para el resto de los índices. Por otra parte, los modelos de análisis funcional se generaron a partir de curvas que fueron ajustadas a modelos no lineales; así mismo, los parámetros de elección del modelo apropiado estuvieron dados por el grado de ajuste del modelo o $\mathrm{R}^{2}$. 


\section{RESULTADOS Y DISCUSIÓN}

Fotosíntesis neta (Pn). La fotosíntesis neta fue influenciada de manera significativa $(\mathrm{P} \leq 0,05)$ por las etapas fenológicas; mientras que las densidades de siembra y la interacción de ambos factores, no mostraron efectos significativos (Tabla 1). Estadísticamente, la mayor Pn se presentó en la etapa vegetativa con $18,94 \mu$ moles de $\mathrm{CO}_{2} \mathrm{~m}^{-2} \mathrm{~s}^{-1}$, la cual, se redujo en un 19,06\% y 19,22\% al inicio y final de la tuberización, respectivamente (Tabla 2). Esta respuesta en ñame espino, puede deberse a la priorización y redistribución de los asimilados al iniciar la tuberización, en detrimento de las tasas de Pn. Al respecto, Archila et al. (1998) y Gaytan et al. (2001), señalan que en la medida que la planta acumula carbohidratos en sus órganos de reserva, las hojas cesan su expansión, y otras inician senescencia, reduciendo su actividad fotosintética. Estos resultados coinciden con los encontrados en yuca, en donde los clones evaluados, mostraron una tendencia a decrecer a partir de la etapa vegetativa (El-Sharkawy, 2007). Sin embargo, en ñame se ha encontrado que el incremento de la tasa fotosintética, es estimulada por los aumentos en la demanda de fotoasimilados del tubérculo (Rodríguez, 2000; Lebot, 2008).

Tabla 1. Cuadrados medios y niveles de significación de la fotosíntesis neta (Pn) en diferentes densidades de siembra y etapas fenológicas del ñame espino.

\begin{tabular}{lcl}
\hline \multicolumn{1}{c}{ Fuente de variación } & gl & \multicolumn{1}{c}{ Pn } \\
\hline Bloque & 2 & 39,6167 \\
Densidad de siembra (A) & 3 & $11,3245 \mathrm{~ns}$ \\
Etapa fenológica (B) & 2 & $52,2634^{* *}$ \\
Interacción A*B & 6 & $1,8617 \mathrm{~ns}$ \\
Error & 22 & 8,7573 \\
$\mathrm{R}^{2}$ & 0,5429 & \\
$\mathrm{CV}(\%)$ & 17,9004 & \\
\hline
\end{tabular}

gl: grados de libertad; Pn: fotosíntesis neta; $\mathrm{R}^{2}$ : coeficiente de determinación; CV: coeficiente de variación. ${ }^{* *}$ Efectos altamente significativos (1\%); ns: no significativo.
Tabla 2. Efecto de las etapas fenológicas sobre la fotosíntesis neta (Pn) en ñame espino.

\begin{tabular}{lc}
\hline \multicolumn{1}{c}{ Etapa fenológica } & Pn $\left(\boldsymbol{\mu m o l e s ~ d e ~} \mathbf{C O}_{2} \mathbf{~ m}^{-2} \mathbf{s}^{-1}\right)$ \\
\hline Vegetativa & $18,94 \mathrm{a}$ \\
Inicio de tuberización & $15,33 \mathrm{~b}$ \\
Final de tuberización & $15,30 \mathrm{~b}$ \\
\hline
\end{tabular}

Promedios acompañados de letras distintas denotan diferencias significativas según la prueba de Tukey (5\%).

Índices de crecimiento. El efecto de las densidades de siembra, solo fue significativo para el IAF (Tabla 3). La TAC mostró una tendencia lineal de orden cuadrático (Figura 1a), en la cual, se evidencia que la máxima tasa de ganancia por planta, inició en la etapa vegetativa, es decir, 34 días después del trasplante (DDT), y se prolongó hasta la tuberización. Según Orkwor (1998), el incremento de la síntesis de biomasa en ñame obedece principalmente a la demanda de asimilados en la formación del dosel y el llenado del tubérculo. El patrón que sigue la ganancia de biomasa de $D$. rotundata, coincide con los reportes de Ñústez et al. (2009), después de evaluar la acumulación y distribución de biomasa de cuatro variedades de papa (Solanum tuberosum L.) bajo las condiciones de Zipaquirá - Colombia, donde estas mostraron una tendencia creciente, que en las últimas etapas se explicó por el llenado del tubérculo.

La TRC se explica por un modelo lineal de orden cúbico. En la etapa inicial se disminuyó la capacidad de producción de biomasa a partir de la existente, pero después de este periodo, aumentó significativamente hasta el inicio de la tuberización (entre 42-94 DDT); a partir de allí se redujo, probablemente por el llenado del tubérculo y la senescencia (Figura 1b). De acuerdo con Rodríguez (2000), los valores descendentes de TRC en la etapa vegetativa obedecen a la dependencia de las plántulas de la reserva de sus semillas. Los incrementos de la eficiencia fotosintética se explicarían por la alta 
demanda de fotoasimilados para la formación del aparato fotosintético en la etapa vegetativa y su posterior reducción, con el llenado del tubérculo y la senescencia. Según Lebot (2008), el compor- tamiento de la TRC obedece a que el crecimiento del tubérculo es rápido en las etapas iniciales del llenado y se reduce en la medida que este proceso culmina.
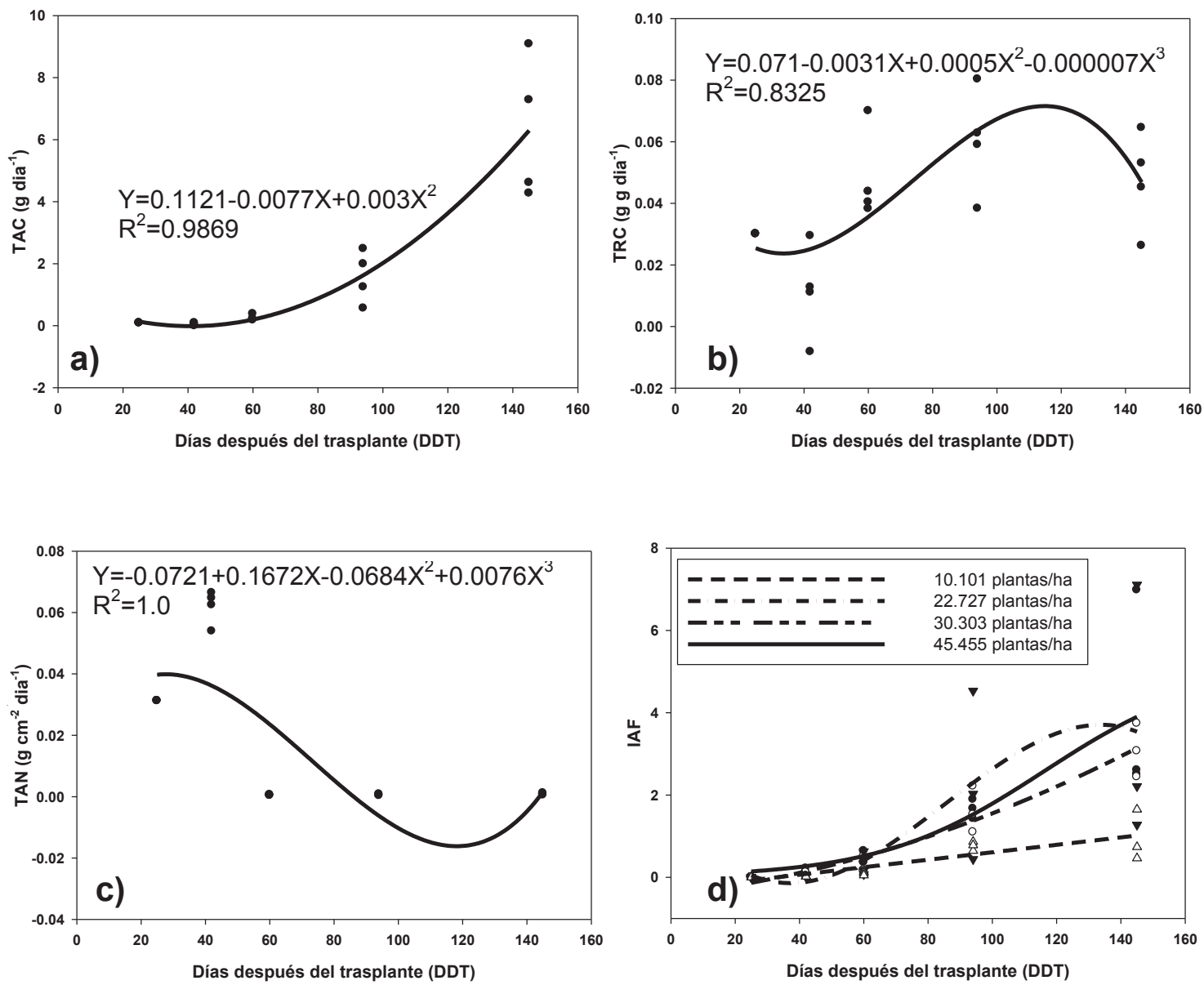

Figura 1. Índices de crecimiento de Ñame Espino (D. rotundata cv. brasilero): a) TAC, b) TRC, c) TAN, d) IAF en función de cuatro densidades de siembra.

Tabla 3. Cuadrados medios y niveles de significación de la prueba de rangos de Friedman para los índices de crecimiento TAC, TRC y TAN, y transformación logarítmica (Y+1) para el IAF, en diferentes densidades de siembra del ñame espino.

\begin{tabular}{lccccc}
\hline \multicolumn{1}{c}{ Fuente de variación } & gl & TAC & TRC & TAN & IAF \\
\hline Bloque & 2 & 0,0000 & 0,0000 & 0,0000 & 0,0659 \\
Densidad de siembra & 3 & $2,3333 \mathrm{~ns}$ & $0,5555 \mathrm{~ns}$ & $0,1111 \mathrm{~ns}$ & $0,0402 *$ \\
Error & 6 & 1,3333 & 2,2222 & 1,9444 & 0,0063 \\
$\mathrm{R}^{2}$ & & 0,4666 & 0,1111 & 0,0277 & 0,8695 \\
$\mathrm{CV}(\%)$ & & 46,1880 & 59,6284 & 55,7773 & 34,1150 \\
\hline
\end{tabular}

ns: no significativo; *: significativo al 5\%. 
Tabla 4. Efecto de la densidad de siembra sobre el IAF al inicio de la tuberización en ñame espino.

\begin{tabular}{cc}
\hline $\begin{array}{c}\text { Densidad de siembra } \\
\text { (Plantas/ha) }\end{array}$ & IAF \\
\hline 10.101 & $0,09 \mathrm{~b}$ \\
22.727 & $0,24 \mathrm{ab}$ \\
30.303 & $0,24 \mathrm{ab}$ \\
45.455 & $0,37 \mathrm{a}$ \\
\hline
\end{tabular}

Promedios con letras distintas de notan diferencias significativas (Tukey; $\alpha=5 \%$ ).

La TAN fue explicada por un modelo del orden cubico (Figura1c). La máxima tasa de asimilación de biomasa por unidad de área y tiempo, ocurrió desde la etapa vegetativa, manifestando una tendencia decreciente en la medida que la planta desarrollaba sus órganos (hasta los 110 DDT aproximadamente), aunque al final del ciclo se incrementó significativamente con el llenado del tubérculo (Figura 1c).

Según Rodríguez (2000), la máxima actividad asimilatoria en la etapa vegetativa obedece a que inicialmente las plantas asignan una alta proporción de asimilados a formación de hojas y tallos; conforme la planta madura, la tasa de particionamiento de asimilados hacia estos órganos decrece significativamente, pero aumenta la que dirige a los tubérculos. En complemento, Gil y Miranda (2007) y Carranza et al. (2009), afirman que esta respuesta se relaciona con el área foliar existente en los primeros días del cultivo, cuyas hojas están más expuestas a la radiación y son más eficientes en la asimilación de $\mathrm{CO}_{2}$, pero el desarrollo de esta disminuye en la medida que aumenta el sombreamiento influyendo en la interceptación de la radiación fotosintéticamente activa y por consiguiente en la fotosíntesis.

El mayor IAF al inicio de la tuberización se alcanzó con la densidad más alta, reduciéndose en pro- medio $36,23 \%$ para 22.727 y 30.303 plantas/ha, y 76,39\% para 10.101 plantas/ha (Tabla 4). A partir de este periodo, el IAF aumentó significativamente (entre 60-145 DDT), donde se observó una tendencia a incrementar en la medida que se aumentan las densidades de siembra, de tal forma que a los 145DDT, el IAF se incrementó en 324,43\% (45.455 plantas/ha), 272,01\% (22.727 plantas/ ha) y $224,76 \%$ (30.303 plantas/ha) con referencia a densidad de convencional de 10.101 plantas/ha (Figura 1d).

La tendencia del área foliar de aumentar en la etapa de tuberización, coincide con lo reportado en papa (Solanum tuberosum L.) bajo condiciones de Zipaquirá - Colombia, donde los máximos incrementos de área foliar, coincidieron con la plena floración e inicio de tuberización para todas las variedades (Santos et al., 2010). Por otra parte, el incremento del IAF ocasionó la reducción de la fotosíntesis desde la tuberización; que según Rodríguez (2000), obedece a nivel de auto-sombreamiento del dosel.

\section{CONCLUSIONES}

Las densidades de siembra evaluadas no influyen sobre la fotosíntesis neta de ñame espino, pero esta tiende a disminuir a medida que avanzan las etapas fenológicas de la planta por el autosombramiento.

El comportamiento de los índices de crecimiento fue igual enlas densidades de siembra, a excepción del IAF, probablemente por la capacidad de la especie de regular su actividad fisiológica en condiciones de competencia.

\section{REFERENCIAS BIBLIOGRÁFICAS}

AGRONET. 2013. Sistema de Estadísticas Agropecuarias.http://www.agronet.gov.co/agronetweb1/ Estad\%C3\%ADsticas.aspx; consulta: febrero, 2015. 
AGUILAR, E. 1998. Guía del Cultivo de Ñame cv. Diamante 22. Ministerio de Agricultura y Ganadería- MAG, Costa Rica. 5p.

ÁLVAREZ, A. 2000. Prácticas Agronómicas para el cultivo de Ñame. En: Guzman, M., Buitrago, G. (ed). Ñame: Producción de Semillas por Biotecnología. Editorial Universidad Nacional de Colombia, Bogotá. 33 - 39p.

ARCHILA, J., CONTRERAS, U., PINZON, H., LAVERDE, H y CORCHUELO, G. 1998. Análisis de crecimiento de cuatro materiales de lechuga (Lactuca sativa). Revista Agronomía Colombiana.16(1):68 - 75.

BALLARÉ, C., SCOPEL, A., y SÁNCHEZ, R. 1995. Plant photo-morphogenesis in canopies, crop growth and yield.Hort- Science. 30(6):1172 - 1181.

BLAKE, R. 1983.A sett size and spacing trial in white yam (Dioscorea alata L. cv.). Memory. Proceedings of the Caribbean Regional Workshop on Tropical Root Crops, Jamaica. 11 - 20 p.

CARDONA, J. 2007. Distancia de siembra en la producción y calidad de ñame Guinea negro (D. rotundata). Journal of agriculture of the University of Puerto Rico. 91(1-2):61 - 65.

CARRANZA, C., LANCHERO, 0., MIRANDA, D. y CHAVES, B. 2009. Análisis del crecimiento de lechuga (Lactuca sativa L.) 'Batavia' cultivada en un suelo salino de la Sabana de Bogotá. Revista Agronomía Colombiana. 27(1): 45.

CONSEJO NACIONAL DE PRODUCCIÓN. CNP. 2015.Analisis de mercados ñame. Boletin №1, Costa Rica, 4 p. Dwelle, B. 1985. Photosyn thesis and photo assimilate partitioning. In: Li, P. (ed). Potato Physiology. Academic Press, INC, Orlando. 35 - 58.

EKANAYAKE, L. y JONG, J. 1992.Stomatal response of some cultivated and wild tuber-bearing potatoes in warm tropics as influenced by water deficits. Annals of Botany. 70(1):53 - 60 .

EL-SHARKAWY, M. 2007. Physiological characteristics of cassava tolerance to prolonged drought in the tropics: Implications for breeding cultivars adapted to sea- sonally dry and semiarid environments. Brazil Journal Plant Physiology. 19(4):257 - 286.

ORGANIZACIÓN DE LAS NACIONES UNIDAS PARA LA ALIMENTACIÓN Y LA AGRICULTURA (FAO). 2013. FAOSTAT. http://www.fao.stat.org. Consulta: agosto, 2015.

GAYTAN, A., PALOMO, A. y GODOY, S. 2001. Eficiencia en la producción y distribución de biomasa en variedades precoces de algodón. Revista Fitotecnia. 24(2): 201 - 202.

GIL, A., MIRANDA, D. 2007. Efecto de cinco sustratos sobre índices de crecimiento de plantas de papaya (Carica papaya L.) bajo invernadero. Revista Colombiana de Ciencias Hortícolas. 1(2):152.

HUNT, R. 1982. Plant growth curves: The functional approach to plant growth analysis. Edward Arnold Publishers, London.

LEBOT, V. 2008.Tropical root and tuber crops.Centre de Coopération Internationale en Recherche Agronomiquepour le Développement, France - London. 229 $-232$.

LIU, C. y ZHONG, C. 2009.Differential response of leaf gas exchange to enhanced ultraviolet-B (UV-B) radiation in three species of herbaceous climbing plants. Acta Ecologica Sinica. 29(2):124 - 129.

MELTERAS, M. 2007. Soil fertility as a factor affecting the production of yams (Dioscorea spp.) in Vanuatu. MPhil Thesis, University of Queensland, Australia.

MINISTERIO DE AGRICULTURA Y GANADERÍA (MAG). 1991. Aspectos Técnicos sobre Cuarenta y Cinco Cultivos Agrícolas de Costa Rica. Boletín técnico No. 74. San José, Costa Rica. 560 p.

ÑÚSTEZ, C., SANTOS, M. y SEGURA, M. 2009. Acumulación y Distribución de Materia Seca de Cuatro Variedades de Papa (Solanum tuberosum L.) en Zipaquirá, Cundinamarca (Colombia). Revista Facultad Nacional de Agronomía. 62(1):4823 - 4834.

ORKWOR, G., ASIEDU, R. y EKANAYAKE, I. 1998. Food Yams: Advances in Research. International Institute of Tropical Agriculture (IITA), Nigeria. 18 p. 
PALENCIA, G., MERCADO, T. y COMBATT, E. 2006. Estudio agrometeorológico del Departamento de Córdoba, Universidad de Córdoba, Montería, 126 p.

REINA, Y. 2012. Documentos sobre economía regional: El cultivo de ñame en el Caribe colombiano. Banco de la República, Cartagena. 6 p.

RODRÍGUEZ, W. 2000. Botánica, domesticación y fisiología del cultivo del ñame (Dioscorea alata). Revista Agronomía Mesoamericana. 11(2):133 - 152.

RODRÍGUEZ, W. y LEIHNER, D. 1997. Growth Rates and Dry Matter Partitioning during different Phenological Growth Stages of the Greater Yam (Dioscorea alata L.). Actes du séminaire international CIRAD-INRA-ORSTOM-CORAF, Montpellier, France. 247 - 249.

SÁNCHEZ, C. y HERNÁNDEZ, L. 1998. Descripción de Aspectos Productivos, de Postcosecha y de Comercialización del Ñame en Córdoba, Sucre y Bolívar. Corpoica - C.I. Turipaná, Cereté, 65 p.

SANTOS, M., SEGURA, M. y ÑÚSTEZ, C. 2010.Análisis de Crecimiento y Relación Fuente-Demanda de Cuatro Variedades de Papa (Solanum tuberosum L.) en el municipio de Zipaquirá (Cundinamarca, Colombia). Revista Facultad Nacional de Agronomía. 63(1):5253 - 5266. 